\title{
Volume Extraction from Body Scans for Bra Sizing
}

\author{
Matteo COLAIANNI ${ }^{* 1}$, Christian SIEGL ${ }^{1}$, Saundra PENKUNAS ${ }^{2}$, Franz ROTT ${ }^{2}$, Günther GREINER ${ }^{1}$ \\ ${ }^{1}$ Computer Graphics Group, University Erlangen-Nürnberg, Germany; \\ ${ }^{2}$ Adidas Group, Germany \\ DOI: 10.15221/15.106 http://dx.doi.org/10.15221/15.106
}

\begin{abstract}
We present a novel method for bra sizing based on surface scan data. While the current standard of finding the proper size for brassieres is based on only two 1-dimensional measurements, our approach takes the entire shape information into account. We propose to use the breast's volume as a good approximation for that shape. To compute the breast volume we introduce a robust and automatic algorithm based on measuring the volume difference of a torso with and without the breasts. Finally, we compare our novel sizing strategy to the traditional sizing of bras. We will show a better distribution across the population of test-subjects. Using the surface scan data in combination with our volume based sizing approach we can furthermore generate mean body shapes for every bra size which is of great benefit for bra development.
\end{abstract}

Keywords: 3D Based Sizing, Volume Extraction, Surface Data Processing, Fit Optimization

\section{Introduction}

\subsection{Motivation}

In the apparel industry the grading and construction of brassieres is a tedious and time consuming process which requires a lot of expert knowledge. The results often are unsatisfying due to an insufficient adaptation to the target population. The current gold standard to determine the cup size of a bra is measuring the difference between the chest and the under-bust girth. This implies that the assumption of equally shaped torsos holds, which in reality is not feasible. Depending on the shape of the body's back, these measurements can be wrong. As a consequence, a proper cup sizing is not always achieved using the traditional standard. Lots of expert knowledge is needed to counteract this problem. We tackle this problem by introducing a paradigm shift in the definition of shape features for the female breast. We argue that a feature closer related to the shape is needed to improve the process of adapting bras to populations. Assuming an almost homogeneous distribution of soft tissue in the region of the breast we propose that the volume is a superior representation for the shape. Thus, we introduce a method for extracting an approximated volume of a breast by only analyzing surface data (i.e. a body scan).

\subsection{Related Work}

The analysis of body shapes for the apparel industry has a wide body of research. Previously a lot of expert knowledge was developed by pattern makers based on anthropometric studies. Some famous basic rules for pattern construction as e.g. introduced by [1] come to mind. Since virtualization technologies found their way into the cloth development process more techniques especially from the field of computational geometry are used in this domain. Morphable Models $([2,3])$ are introduced to describe geometry as a statistic over vertex positions. They describe a shape without the need of measurements and can be used for statistical shape analysis and body synthesis. Hasler et al. [4] extended the idea of body shape statistics by labelling the feature space with additional values (e.g. weight, body height). Since the presented work analyzes surface data acquired from body scans, a suitable representation of the shape has to be generated. Watertight manifold triangle meshes are required by many algorithms dealing with the manipulation and analysis of geometry. A lot of work exists on approximating scattered point data by a triangle mesh template $([5,6])$. Rödel et al. [7] introduced a report on shape based analysis of female torsos. We introduce a two-step approach for fitting scan data with a mesh inspired by Zollhöfer et al. [8]. They used a statistical model as a prior and introduce additional constraints for an optimization of the result.

To our knowledge no related work exists that achieve the extraction of cup volume approximation based only on the surface information.

\footnotetext{
* matteo.colaianni@fau.de; +49- 9131- 85-29923; www9.cs.fau.de
} 


\subsection{Overview}

In this work, we present a lightweight and easy to implement method for extracting the breasts' volume using only an upper body surface scan. To achieve this, we measure the volume difference of the original scan and an adapted version without the breasts. For the virtual removal of the breasts we present a robust automatic feature detector that locates the region of interest. Subsequently we use a smoothing approach to find an upper body representation without breasts. We use this new method of volume extraction to re-cluster a population of females and the results are compared to the traditional way of sizing. We show that our method leads to better distributed size groups. Finally, we present the representative shapes for the clusters and outline how they can be used in pattern construction for brassieres.

\section{Method}

\subsection{Preprocessing of Scanned Data}

With the presented method the volume of a woman's breast can be extracted from a single body scan of the torso. For this purpose the raw scan data have to be transferred into a mesh representation, as the used surface deformation scheme works on manifold surfaces. For extracting a mesh representation we use a two-step approach. In the first step, we register a coarse statistical model to the point cloud (see [10]). To further refine the mesh, non-rigid registration based on $[6,11]$ is performed. The advantage of this registration method is to preserve semantic landmarks on the mesh relative to the human body.

It is a common practice in apparel construction to analyze symmetric body shapes. Therefore, we symmetrize the meshes aligned to their principal axis.

\subsection{Automatic Extraction of the Feature Region}

The presented method works on female body scans as input data and assumes the volume to be the difference between a torso shape and a breast-less version of the torso. In order to generate a torso without the breasts we need to isolate the appropriate surface region. Including the whole mesh into the computation described in the following section will lead to incorrect results. To label the proper region around the cups a robust and automatic feature detection scheme is implemented. The method first extracts the height of the under-bust (see Figure 1). For this, arbitrarily fine axial plane cuts with the mesh are analyzed in ascending direction. For each plane, we determine a point behind the middle of the cup - we refer to it as the origin. From this point we shoot rays to intersect the front of the torso contour (see Figure 1, right). The ray with the most extent within a certain angle is chosen to be the feature value of the current plane cut. Now, every height has its own representing feature value. The function over the height is depicted in Figure 2, left. We are able to find the height of maximum curvature by evaluating the second derivative of the function (Figure 2, right). This corresponds to the under-bust height.
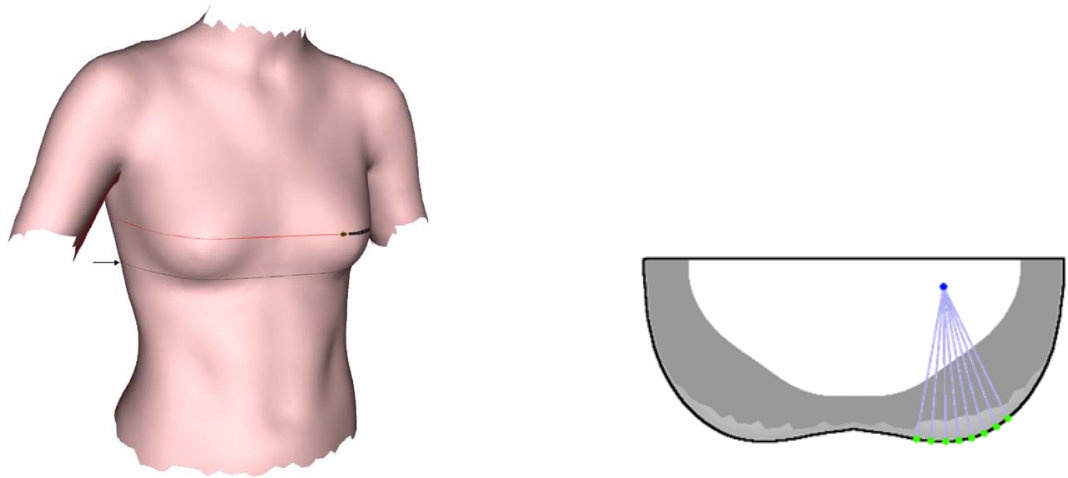

Figure 1, left: The torso with the marked under-bust location (grey contour). Right: The maximum extent is found by sampling points on the breast surface. 

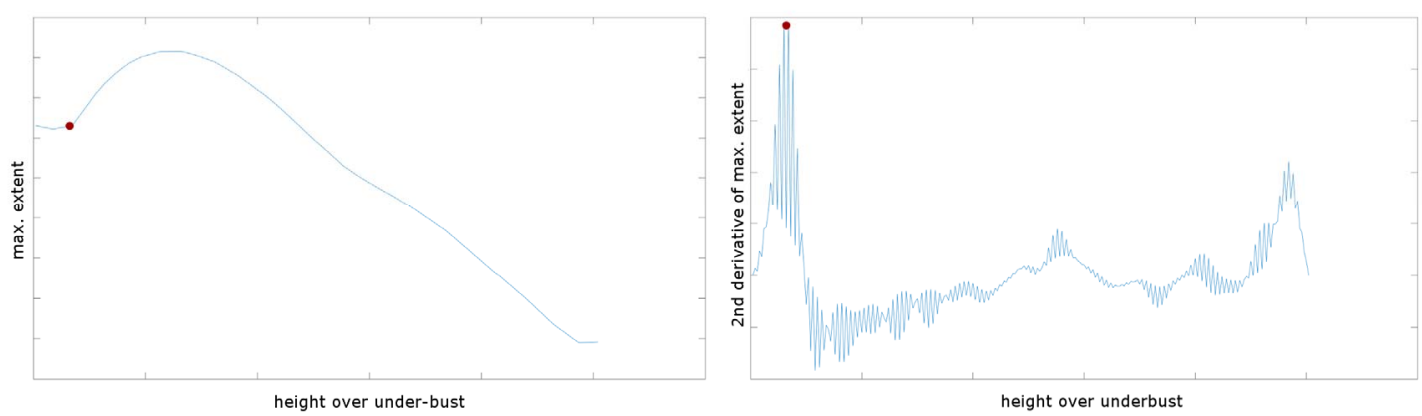

Figure 2, left: The maximum extent for the front contour along each slice, the shape of the breast is visible in the function values. Right: The $2^{\text {nd }}$ derivate of the discrete maximum extent (left). The first peak denotes the under-bust position.

The region of interest finally is lower bounded by the height of the under-bust-girth. The upper bound is defined by a semantic landmark on the body. In addition only vertices with a front facing normal are included in the smoothing (see Figure 3).

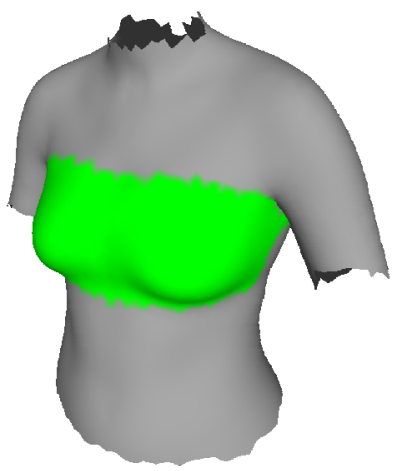

Figure 3: The automatically extracted breast region. Within this area the mesh is deformed (see section 2.3).

\subsection{Cup Volume Approximation}

After the extraction of the feature region, the volume of the breast has to be extracted. The only input our method needs is the surface information. Since we assume a homogenous distribution of the soft tissue within the feature region, the cup volume approximation is calculated by

$$
\tilde{V}_{\text {Cup }}=V_{\text {Torso }}-\tilde{V}_{\text {Cupless }} \text {. }
$$

Extracting $V_{\text {Torso }}$ from the original upper body surface scan is straight forward. Computing the volume of the breast-less instance is more involved. The region of the breast has to be processed in a way that preserves the torso while removing the breasts. This information is not part of the scan. Furthermore, no ground truth data exists. There is no standard for defining which part of the female breast belongs to the part that later will be supported by the bra. For this reason we defined our own algorithm that generates an upper body mesh representation without the breasts.

We use a Laplace-Beltrami operator defined for discrete surfaces (as meshes) to smooth the formerly extracted region of interest (see section 2.2). The method iterates over each vertex $x_{i}$ of the mesh multiple times and replaces it by the average of its neighboring vertices:

$$
\Delta_{M} x_{i}=\frac{1}{\left|N\left(x_{i}\right)\right|} \sum_{x_{j} \in \mathcal{N}\left(x_{i}\right)}\left(\mathrm{x}_{\mathrm{j}}-\mathrm{x}_{\mathrm{i}}\right)
$$

with $\mathcal{N}\left(v_{i}\right)$ being the 1-neighbourhood of a vertex $v_{i}$. Iteratively the mesh is smoothed and high shape features (such as breasts) disappear. Note, smoothing the entire mesh would lead to incorrect results, for this reason we apply the presented Laplace-Beltrami operator only on the formerly extracted breast-region (see Section 2.2). The original Shape and the smoothened instance are shown in Figure 4. 

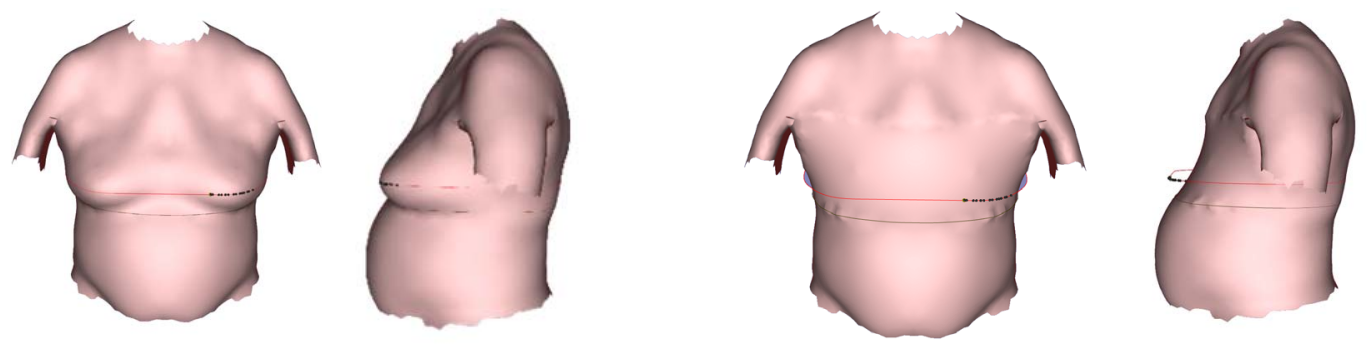

Figure 4: The original torso shape (left) leads to a smoothed instance without the cups (right).

\section{Result}

\subsection{Traditional Measurement-Based Clustering}

The main criterion of the developed method's quality is its influence on bra sizing. For evaluation we use 1183 meshes generated based on the CAESAR database [9]. First, we used the current approach, measuring the difference between under-bust and chest girth. The meshes then were separated into the traditional under-bust groups. Given the relatively small number of scanned subjects, we used four under-bust sizes (see Table 1).

\begin{tabular}{|c|c|c|}
\hline Cluster & Lower Boundary & Upper Boundary \\
\hline 70 & $-\infty$ & 74.9 \\
\hline 80 & 75.0 & 84.9 \\
\hline 90 & 85.0 & 94.9 \\
\hline 100 & 95.0 & $+\infty$ \\
\hline
\end{tabular}

Table 1: Boundaries for the under-bust clusters (in $\mathrm{cm}$ ).

The distribution is shown in Figure 5. Here the majority of subjects fall into the cluster 80 . Next, the clusters have been separated into the traditional cup-sizes (compare Table 2).
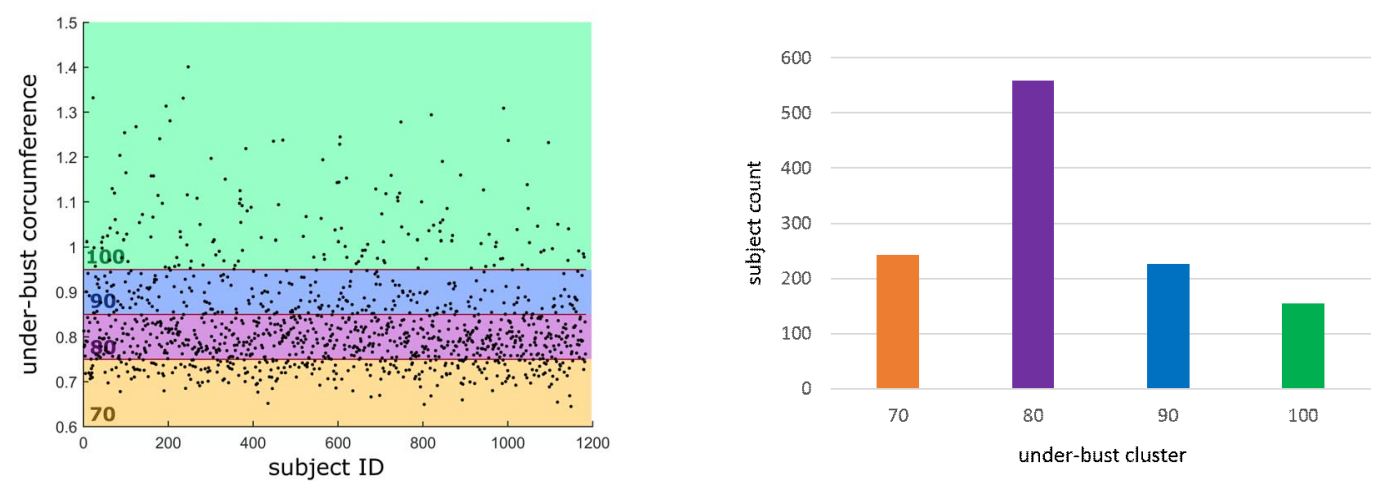

Figure 5: Distribution of the scan data according the under-bust circumference.

\begin{tabular}{|l|c|c|c|c|c|c|c|}
\hline & $<A$ & A & B & C & D & E & >E \\
\hline Lower Boundary & $-\infty$ & 12.0 & 14.0 & 16.0 & 18.0 & 20.0 & 22.0 \\
\hline Upper Boundary & 11.9 & 13.9 & 15.9 & 17.9 & 19.9 & 21.0 & $+\infty$ \\
\hline
\end{tabular}

Table 2: Boundaries for the cup sizes. Measured as delta between the chest and the under-bust girth (in $\mathrm{cm}$ ).

The overall result of the traditional clustering can be seen in Figure 6 . This method makes the assumption that the cup shape is entirely represented by only two measurements (chest and under-bust circumference). If this assumption holds, the cup sizes should be normally distributed. Analyzing the probability function of cup sizes in Figure 6 shows that this is not the case. We therefore replace it by the presented volume based approach. This leads to an improved distribution (see Figure 8) as well as a more suitable description for the definition of female breast shapes. 


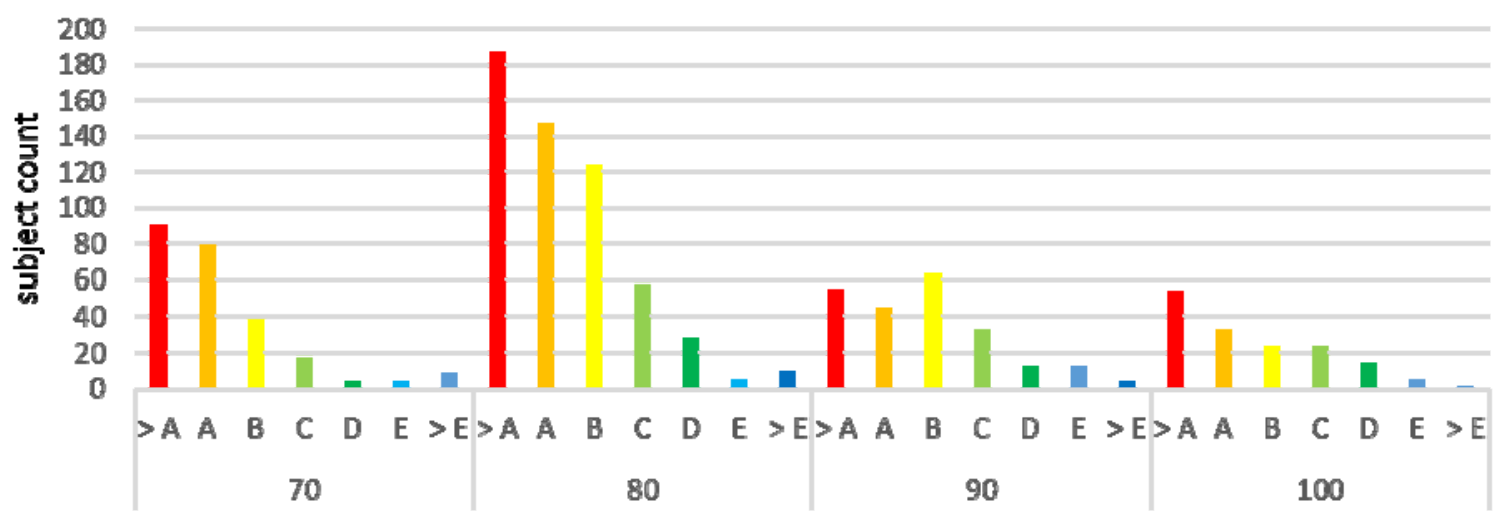

under-bust and cup clusters

Figure 6: Distribution of the scan data according the traditional combined sizing.

\subsection{Volume-Based Clustering}

The presented breast volume approximation now is used to replace the traditional cup sizing. While the subjects are still grouped according to their under-bust circumference, the two-measurement based cup sizing is replaced by volume clusters. We introduce five volume clusters V1-V5. Every subject from the database was evaluated according to its breast volume. Afterward, we searched for the best cluster bounds using the $k$-means method. This commonly used method separates a set of samples into clusters while ensuring each sample to have the minimum distance to its subset-center (for details, see [12]). Note, that the method is neither limited to 5 volume clusters nor constrained to have the same amount of volume group for each under-bust cluster. Our experimental clustering is shown in Table 3 while Figure 7 shows the boundaries graphically to gain intuition. Note, that each under-bust cluster has its individual bounds for the volume clusters.

\begin{tabular}{|c|c|c|c|c|c|c|}
\hline & bound & V1 & V2 & V3 & V4 & V5 \\
\hline \multirow{2}{*}{70} & Min & $-\infty$ & 635.90 & 833.50 & 1045.30 & 1334.60 \\
\cline { 2 - 7 } & Max & 635.89 & 833.49 & 1045.29 & 1334.59 & $+\infty$ \\
\hline \multirow{2}{*}{80} & Min & $-\infty$ & 778.60 & 1045.60 & 1327.90 & 1663.60 \\
\cline { 2 - 7 } & Max & 778.59 & 1045.59 & 1327.89 & 1663.59 & $+\infty$ \\
\hline \multirow{2}{*}{90} & Min & $-\infty$ & 1049.20 & 1364.00 & 1699.30 & 2138.01 \\
\cline { 2 - 7 } & Max & 1049.19 & 1363.99 & 1699.29 & 2138.00 & $+\infty$ \\
\hline \multirow{2}{*}{100} & Min & $-\infty$ & 1780.70 & 2310.80 & 2948.30 & 3890.60 \\
\cline { 2 - 7 } & Max & 1780.69 & 2310.79 & 2948.29 & 3890.59 & $+\infty$ \\
\hline
\end{tabular}

Table 3: Boundaries for the cup volume groups per under-bust cluster (in ccm).

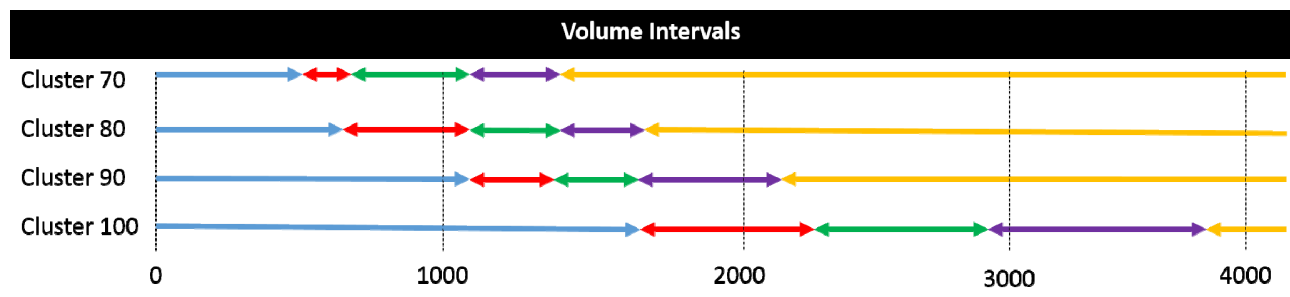

Figure 7: Boundaries for the cup volume (V1, V2, V3, V4, V5).

Figure 8 shows the distribution of subjects using the adapted approach. Note the substantially better distribution of the subjects within as well as amongst all under-bust clusters. 


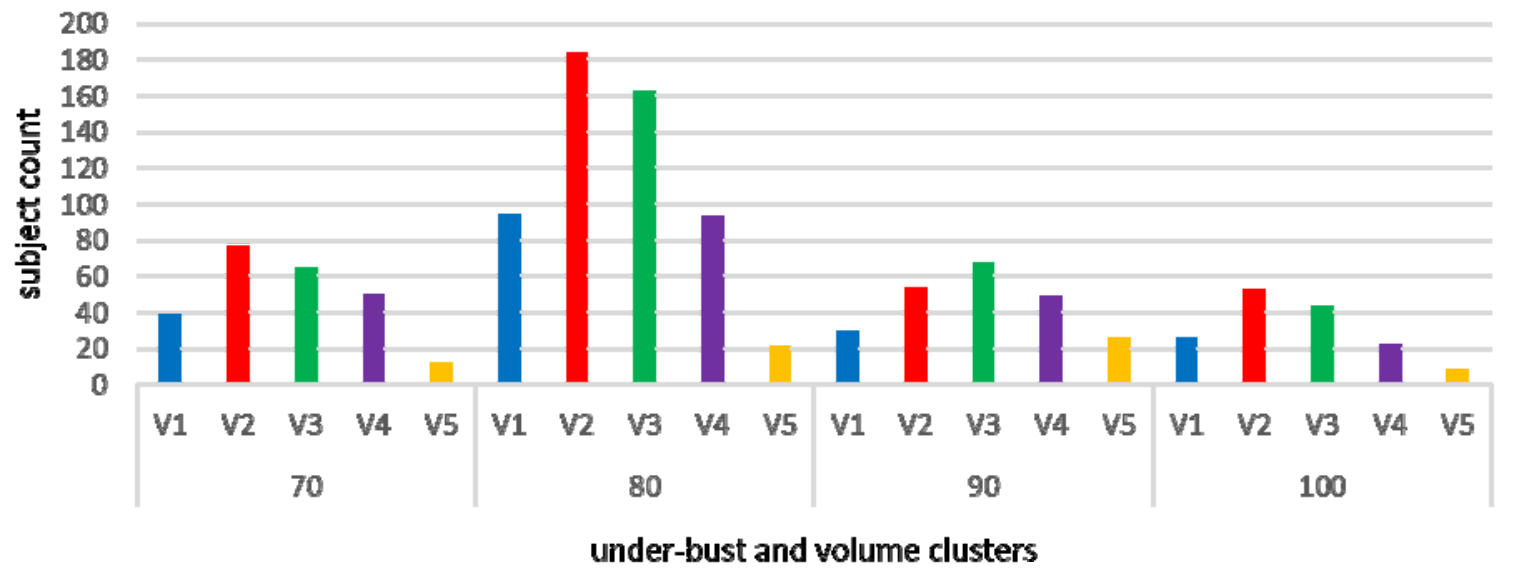

Figure 8: Distribution of the data using the volume based sizing. Note the more equally distributed samples compared to Figure 6.

One additional key difference to the static cup sizing rule of the traditional approach is that our volume clusters adapt to each under bust cluster. For example a V4 cup for cluster 90 covers approximately the same volume range as a V2 cup for cluster 100 (see Figure 7). The traditional approach applies one rule for cup sizes over all subjects - the difference between two measurements. This leads to the second reason for the better distribution. The shape of the cup itself is not approximated well with the measurement-based approach.

Figure 9 shows the redistribution of sample subjects from under-bust cluster 80 . For example, the marked subject was formerly classified as an A-cup. In reality, the woman exhibits a cup volume of V5 which highly disagrees with an A-cup classification.

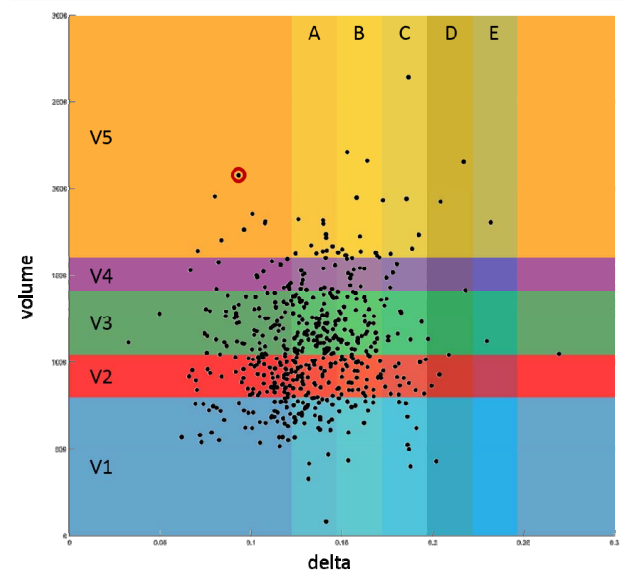

Figure 9: Redistribution of sample subjects from under-bust cluster 80 . The red labelled subject holds a high mismatch between the traditional and the presented method of size definition.

With our volume based approach and the underlying measured 3D geometry we resemble the population more closely. Additionally we can generate mean breast shapes for every cluster. This is an excellent basis for the pattern creator of brassieres.

\section{Applications}

\subsection{Sizing for a Given Population of Scans}

One previously mentioned fundamental advantage of our new volume based approach to clustering female breasts is the additional supply of mean shapes. In the following we will show these representative torso shapes that closely resemble the population of our scan data. These generated average shapes can be used for measurement, analysis and of course pattern construction. We used the previously shown distribution (Figure 8) of under-bust clusters with five volume groups per cluster. 
Using these clusters, we synthesized average shapes for each group as shown in Table 4. Using our approach in combination with the shown distribution graphs one can decide for a sizing strategy for brassiere collection and reorganize the number of groups within the under-bust clusters.

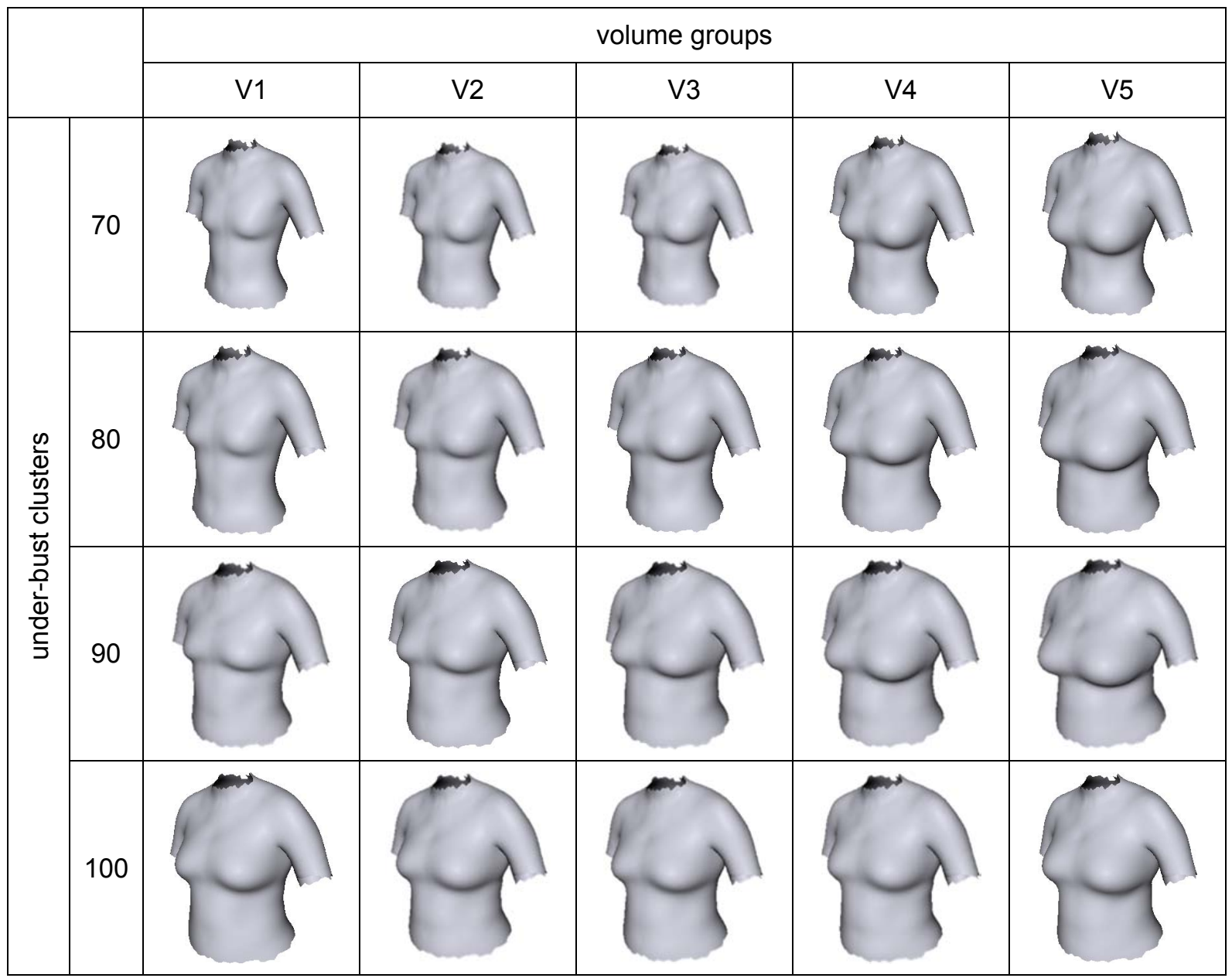

Table 4: Synthesized Average Shapes for the proposed under-bust clusters and breast volume groups.

\subsection{Brassiere Construction on 3D Avatars}

Having the synthesized shapes at hand, the virtual assisted development of a constructed bra is possible. We sketch a system, where cuts can be applied directly on the virtual model (e.g. [13]). After cutting the shape from the surface, a flattening method (see [14]) leads to a good approximation to the shape of the final pattern. Figure 10 shows the use case where a synthesized average shape is directly used to apply cuts for further processing.
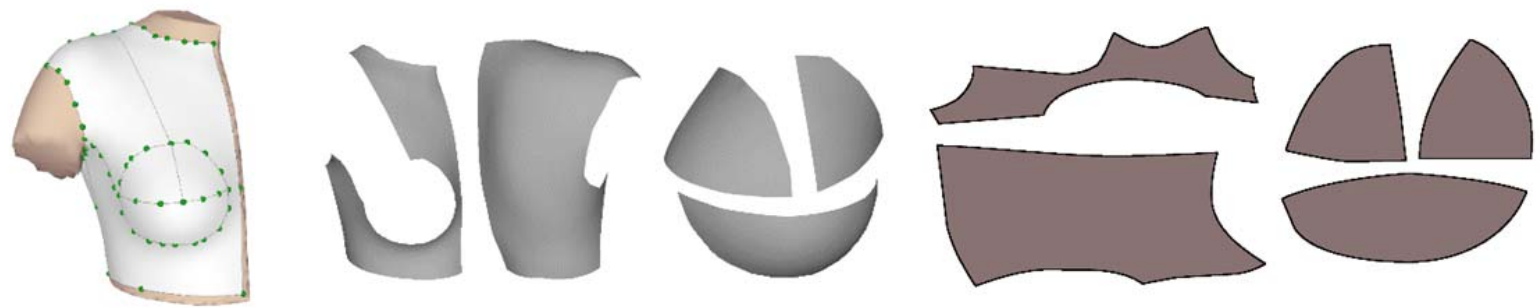

Figure 10: Virtual brassiere construction pipeline. The style is applied to the synthesized torso (left), the patches are cut (middle) and a flattening scheme leads to samples. With those, a pattern can be developed (right). 


\section{Limitations}

Even though the presented method of extracting chest volumes for sizing yields good results, some limitations of the system have to be discussed. The main issue is the lack of a ground truth test to evaluate the proposition of extracting the volume. In practice this evaluation is not possible due to obvious reasons. Even with comparable volumetric data from medical imaging the proper segmentation of the breast region is ambiguous. Another disadvantage of introducing volume clusters is the lack of intuition for this measurement. While taking the two measurements needed for the traditional approach is simple, the volume cannot be extracted with the usual measuring tape. With the growing importance of commodity body scanning hardware in the retail domain, there will be various possibilities to integrate a simple volume approximation system for breasts into a store. This way women can profit from our novel sizing system that enhances the quality of bra fit.

\section{Conclusion and Outlook}

We have presented a novel method for extracting the volume of a female breast from a 3D surface scan. Using this algorithm we have introduced a sizing scheme for bras that showed to outperform the current gold standard for bra sizing in terms of reproducing the sample population. Additionally to this new sizing scheme we are able to supply the industry with mean torso shapes of every new bra size. Using these sample shapes, the development of the actual bra is greatly simplified and less expert knowledge from the bra designer is required.

\section{References}

[1] M. Müller. Der Zuschnitt Für Die Damenschneiderei - Blusen, Kleider und Wäsche. Deutsche Bekleidungs-Akademie München,1962.

[2] Volker Blanz and Thomas Vetter. A morphable model for the synthesis of $3 \mathrm{~d}$ faces. In Proceedings of SIGGRAPH '99, pages 187-194, 1999.

[3] Brett Allen, Brian Curless, and Zoran Popovi'c. The space of human body shapes: Reconstruction and parameterization from range scans. ACM Trans. Graph., 22(3):587-594, July 2003.

[4] N. Hasler, C. Stoll, M. Sunkel, B. Rosenhahn, and H.-P. Seidel. A statistical model of human pose and body shape. In Computer Graphics Forum (Proc. Eurographics 2008), volume 2, 2009.

[5] Brian Amberg, Sami Romdhani, and Thomas Vetter. Optimal step nonrigid icp algorithms for surface registration. In Proceedings of CVPR'07, pages 1-8, 2007.

[6] Michael Zollhöfer, Ezgi Sert, Günther Greiner, and Jochen Süßmuth. Gpu based arap deformation using volumetric lattices. In Eurographics (Short Papers) 2012, pages 85-88, 2012.

[7] H. Rödel. Entwicklung parametrischer virtueller Formkörper weiblicher Personen zur dreidimensionalen Konstruktion von körpernaher Bekleidung und Miederwaren, Final Report. TU Dresden, 2008.

[8] M.Zollhöfer, J.Thies, M. Colaianni, M. Stamminger, G. Greiner. Interactive Model-based Reconstruction of the Human Head using an RGB-D Sensor. In Journal Computer Animation and Virtual Worlds (25, Nr. 3-4, pages 213-222), 2014.

[9] K.M. Robinette, S. Blackwell, H. Daanen, M. Boehmer, S. Fleming. Civilian American and European Surface Anthropometry Resource (CAESAR), Final Report. Volume 1. Summary. AFRL-HE-WP-TR-2002-0169, Human Effectiveness Directorate, Crew System Interface Division, $2255 \mathrm{H}$ Street, Wright-Patterson AFB OH 45433-7022 and SAE Irternational, 400 Commonwealth Dr., Warrendale, PA 15096.

[10]M. Colaianni, M. Zollhöfer, J. Süßmuth, B. Seider, G. Greiner. A Pose Invariant Statistical Shape Model for Human Bodies. Proceedings of the 5th International Conference on 3D Body Scanning Technologies, 2014.

[11] Olga Sorkine and Marc Alexa. As-rigidas-possible surface modeling. In Proceedings of SGP'07, pages 109-116, 2007.

[12] J.B. MacQueen. Some Methods for classification and Analysis of Multivariate Observation. In Proceedings of 5th Berkley Symposium on Mathematical Statistics and Probability, 1967.

[13] Optitex 3D Creator [Computer Software], 2015

[14]Y.L. Choi, Y.Nam, K.M. Choi, M.H. Cui. A Method for Garment Pattern Generation by Flattening 3D Body Scan Data. In Digital Human Modeling, Volume 4561, Springer Berlin Heidelberg, 2007. ISBN: 978-3-540-73318-8 